\title{
EXPANSIÓN MAXILAR EN LA CORRECCIÓN DE MORDIDA CRUZADA POSTERIOR DERECHA.
}

\section{MAXILAR EXPANSION IN CORRECTION OF RIGHT POSTERIOR CROSS-BITE}

\author{
Zambonino-Palma Paulina ${ }^{1}$ Gurrola-Martinez Beatriz ${ }^{2}$ Casasa Araujo Adán*3 \\ ${ }^{1}$ Residente de segundo año de la maestría de Ortodoncia y Ortopedia Maxilofacial del Centro de Estudios Superiores de \\ Ortodoncia, CESO.México. \\ ${ }^{2}$ Profesora del CESO y profesor de tiempo completo "Titular C" de la carrera de Cirujano Dentista de la Facultad de \\ Estudios Superiores Zaragoza. UNAM.México. \\ ${ }^{3}$ Director del CESO.México. \\ *adancasasa@gmail.com
}

\section{Resumen}

\begin{abstract}
OBJETIVO:Se presenta un paciente de 11 años de edad, en el análisis intraoral se diagnosticó como: paciente clase I esquelética, crecimiento normodivergente, clase II molar derecha, clase I molar izquierda, clase II canina bilateral, mordida cruzada en los dientes $12 ; 22 ; 24$ y 25 ; y posterior derecha, apiñamiento severo superior y leve en inferior. El tratamiento consistió en: Expansión transversal, Alineación, nivelación, stripping, detallado, ratención, la aparatología utilizada Expansor con tornillo tipo Hyrax (9 mm) inferiores brackets prescripción Roth 0,22 x 0,28; bandas en los molares 6's superiores, tubos bondables en los molares 16, 26; superiores y los 17 y 27; superiores e inferiores. La retención estuvo a cargo de un retenedor circunferencial con cinturón vestibular superior, y Hawley inferior con cinturón vestibular.
\end{abstract}

Palabras clave: mordida cruzada posterior derecha.

\begin{abstract}
AIM: An 11-year-old patient is presented. In the intraoral analysis, it was diagnosed as: skeletal class I patient, normodivergent growth, right molar class II, left molar class I, bilateral canine class II cross-bite in 12; 22; 24 and 25; and right posterior, superior and mild severe crowding in inferior. The treatment consisted of: Transverse expansion, Alignment, leveling, stripping, detailed, ration, equipment Used Expander with screw type Hyrax $(9 \mathrm{~mm})$, lower brackets prescription Roth 0,22 $\times$ 0,28; bands in the molars 6's upper, curves bondable in the upper 16; 26; and upper 17; 27; and inferiors The retention was a load of a circumferential retainer with superior vestibular belt, and inferior Hawley with vestibular belt.
\end{abstract}

Key words: right posterior crossed bite.

\section{INTRODUCCIÓN}

Espasa señala que la consecuencia de las dimensiones transversas reducidas de la arcada superior se traduce en una mordida cruzada posterior, y diferentes manifestaciones clínicas que varían de la mordida cruzada unilateral, diagnosticada en el $90 \%$ de los casos de mordida cruzada posterior, a la mordida cruzada bilateral o completa, donde la mandíbula contiene totalmente al maxilar. Observándose un porcentaje en el desarrollo de los estadios tempranos oclusal del 5 al $7 \%$, hasta el 22 y $23 \%$, al corregir la mordida cruzada posterior se logra un mayor número de relaciones transversas normales, esto con la presencia de los molares definitivos. ${ }^{1}$

Por otra parte Beraud menciona que el origen de la mor- dida cruzada es desconocido, puede ser: esquelética, dental y funcional. La esquelética es la discrepancia en la estructura ósea mandibular o maxilar superior, identificándose por una desigualdad básica en el ancho de las arcadas. La cruzada dental es la consecuencia del patrón de erupción; o que no haya irregularidad del hueso basal. Y la mordida cruzada funcional es el desplazamiento de la mandíbula a una posición anormal, pero a menudo más descansado. ${ }^{2}$ Un problema frecuente en la población son las maloclusiones transversales ya sea por índole genéticas, ambientales Castañer dice que en los jóvenes, de grandes ciudades se ve cada vez más la presencia de problemas respiratorios, alimenticios. ${ }^{3}$ Basándonos en la clasificación de las maloclusiones transversales 

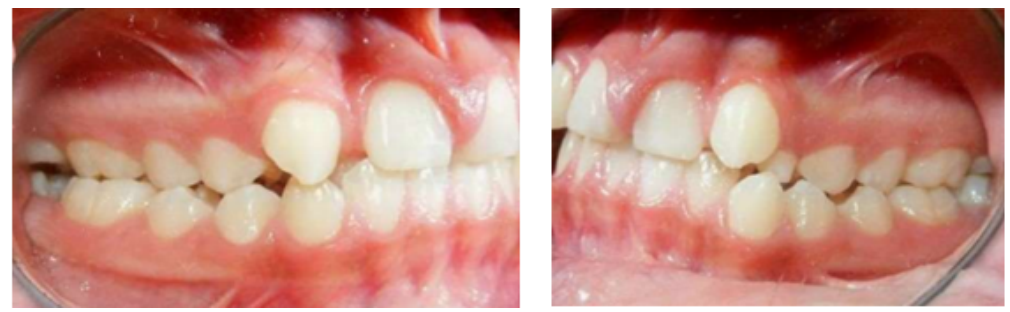

Fig. 1. Lateral Derecha e Izquierda.
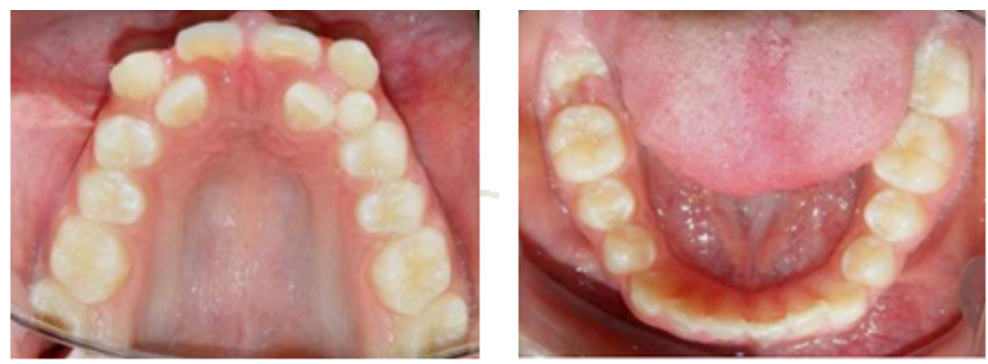

Fig. 2. Oclusal Superior e Inferior

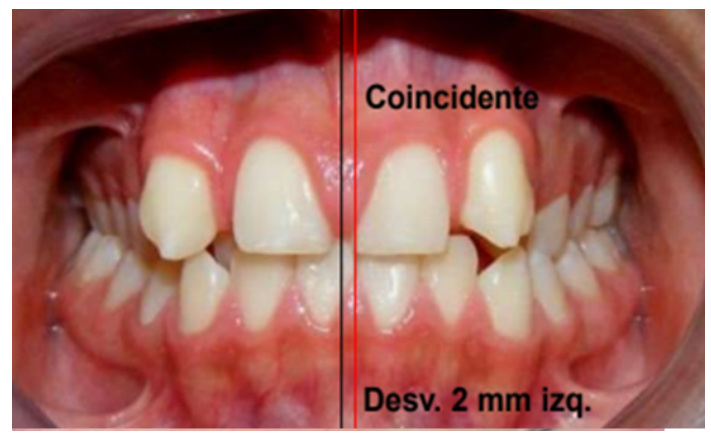

Fig. 3. Frente.

propuesta por Lorente en el año 2002, tabla 1 se ordenan en mordidas cruzadas unilaterales, utilizando esta clasificación presentaremos las alternativas terapéuticas para cada una de ellas.

Una vez tenemos las mordidas cruzadas transversales clasificadas debemos elegir el tratamiento de expansión más adecuado. La expansión maxilar como tratamiento de la discrepancia óseo-dentaria, ha sido el tratamiento ortodontico Los primeros en diseñar aparatos para expansión fueron Coffin para expansión del maxilar, Angell el que habría la sutura palatina media. Sin embargo fue en el inicio del siglo $\mathrm{XX}$, que se reportó sobre las expansiones, las cuales sólo producían cambios a nivel dentoalveolar, sin cambios esqueléticos. Hasta que Haas estudio sobre los efectos dentales como esqueléticos de la expansión rápida maxilar observando que se producía la apertura de la sutura palatina media, un desplazamiento del maxilar hacia delante y abajo y una posterorrotación mandíbular.

Realizado estudios para prevenir los efectos indeseables de la expansión rápida, para evitar la posterrotación mandibular. El aparato ortodóncico Quad-Helix es el tratamiento que consigue la compresión dentaria con fuerzas suaves y prolongadas según Ricketts. Por otra parte autores como: Greenbaun y Zachrisson trabajaron los efectos de la expansión en los tejidos periodontales.

Respecto al Quad-Helix en 2005 Giron de Velasco comprobó el efecto de la expansión con él era sobre todo dental encontrando la extrusión del molar superior. Pero si la expansión se realizaba con un disyuntor descartando las compensaciones dentales, la expansión era esquelética y se producía una intrusión del molar superior. ${ }^{4}$

Ortopédicamente la expansión maxilar funciona con separar el paladar a nivel de la sutura media, por la compresión del maxilar superior, los que apoyan este procedimiento 


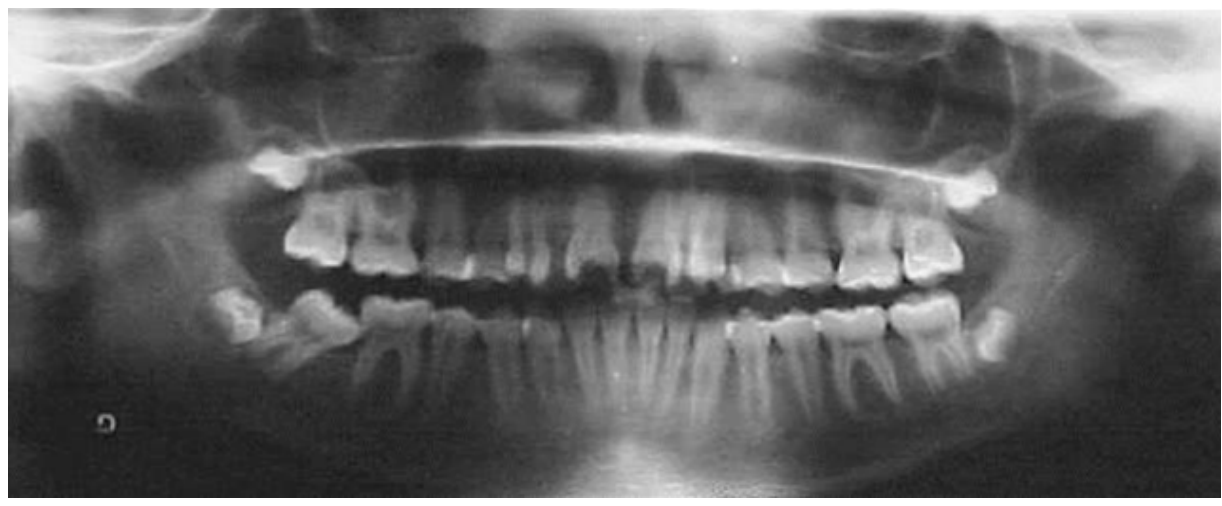

Fig. 4. Radiografía Panorámica.
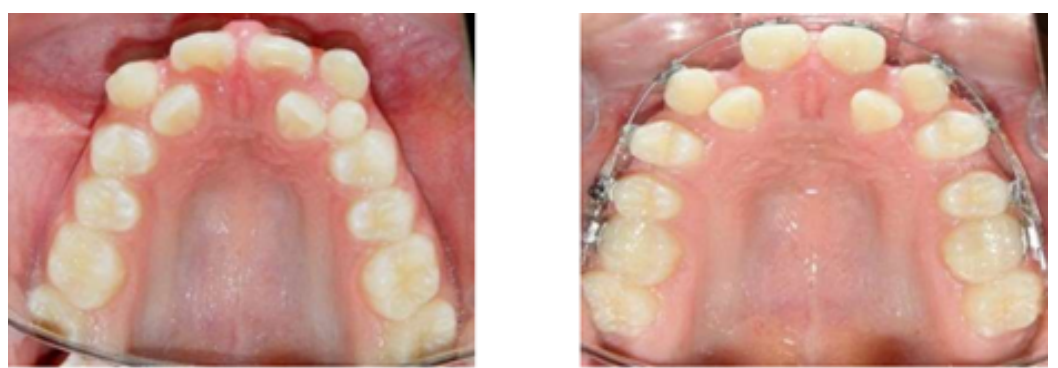

Fig. 5. Comparación Oclusal Superior Inicio-Progreso.

tienen el fundamento teórico que aplicando una fuerza rápida a los dientes posteriores, no habrá tiempo suficiente para que éstos se inclinen y que la fuerza se transferirá a la sutura, que se abrirá mientras los dientes se desplazan de forma mínima, por día la separación lograda es de 0,2 a $0,5 \mathrm{~mm}$ y resulta un incremento intermolar hasta de $8 \mathrm{~mm}$. Efectos sobre el complejo maxilar. La ERM ocurre cuando las fuerzas aplicadas a los dientes y procesos alveolares maxilares exceden los límites necesarios para el movimiento dental ortodóncico. La presión aplicada actúa como una fuerza ortopédica que abre la sutura media palatina. En los procesos alveolares se provoca una compresión del ligamento periodontal que inclina el anclaje dental y una apertura gradual de la sutura media palatina. Oclusalmente es mayor en el sector anterior (incisivos) que en el borde posterior de la sutura (molares) $\mathrm{y}$ con un movimiento hacia abajo y adelante del punto A (generalmente mínimo).

Efectos sobre los procesos alveolares. La resilencia del hueso, permite la inclinación de los procesos ocurre temprano durante la ERM, la mayoría de las fuerzas aplicadas tienden a disiparse dentro de 5 a 6 semanas y una vez terminada la estabilización. Por otro lado los efectos dentarios: el maxilar superior: es dos veces mayor a nivel de los incisivos centrales que a nivel de los molares la separación. Las fibras elásticas transeptales unen las coronas de los incisivos rápidamente, y sólo al cabo de unos cuatro meses logran la convergencia de sus raíces. Se puede observar una ligera extrusión y palatinización de los incisivos centrales. En el maxilar inferior se ve un ligero enderezamiento o la permanencia del eje axial de los molares, tendencia a la rotación hacia abajo y atrás debido a la inclinación y extrusión de los molares superiores. En la anchura de la cavidad nasal anatómicamente hay un aumento, de modo especial en el piso de la nariz región anteroinferior de las fosas nasales. Es importante saber que si la obstrucción del paso de aire se encuentra más posterosuperior será más difícil de corregirla con la ERM. La cavidad nasal se amplía en un promedio de $1,9 \mathrm{~mm}$, y a nivel de los cornetes inferiores de 8 a $10 \mathrm{~mm}^{5}$

Por otra parte, es una indicación de la expansión ortopédica del paladar cuando hay contracción del diámetro transversal de la arcada superior en niños y jóvenes de 15-16 años, para corregir una discrepancia transversal de más de $5 \mathrm{~mm} .{ }^{6}$ El tratamiento preventivo de la mordida cruzada posterior unilateral funcional en la dentición mixta es importante para la ortodoncia, ya que provee el correcto posicionamiento de bases oseas, dientes, de la articulación temporomandibular, cuando el sistema estogmatognatico está en pleno crecimiento y desarrollo. ${ }^{7}$

\section{REPORTE DE UN CASO}

Se reporta el caso clínico de una paciente de 11 años. El paciente refiere que acude a consulta porque desea una 

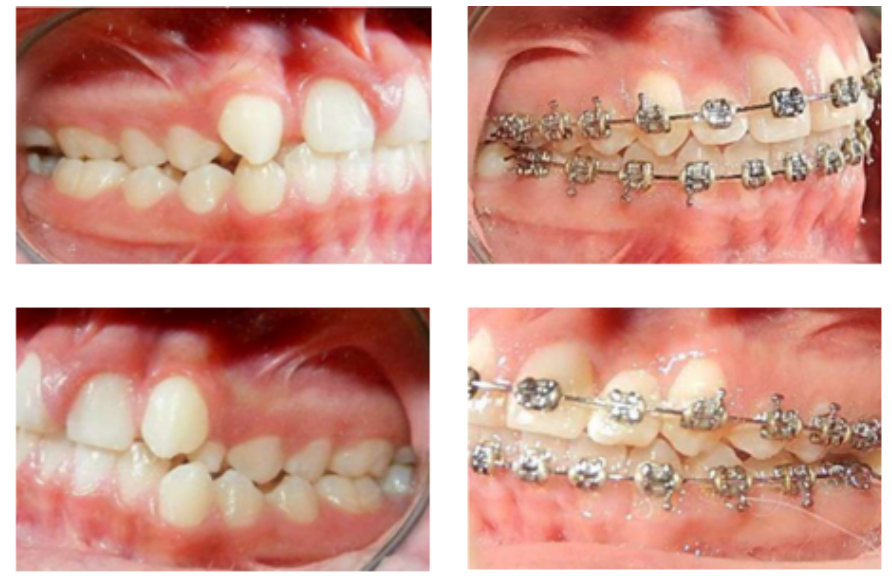

Fig. 6. Comparación Lateral Derecha, Lateral Izquierda Inicio-Progreso.
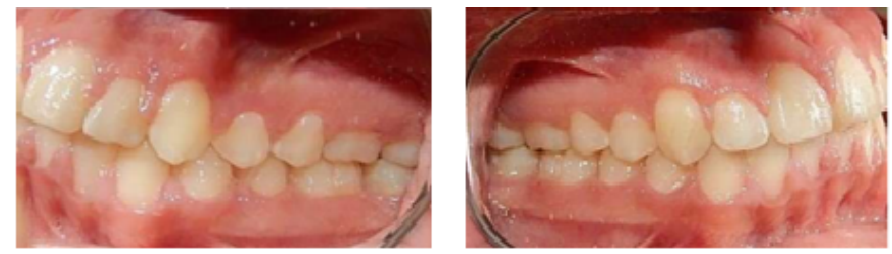

Fig. 7. Final Lateral Derecha, Lateral Izquierda
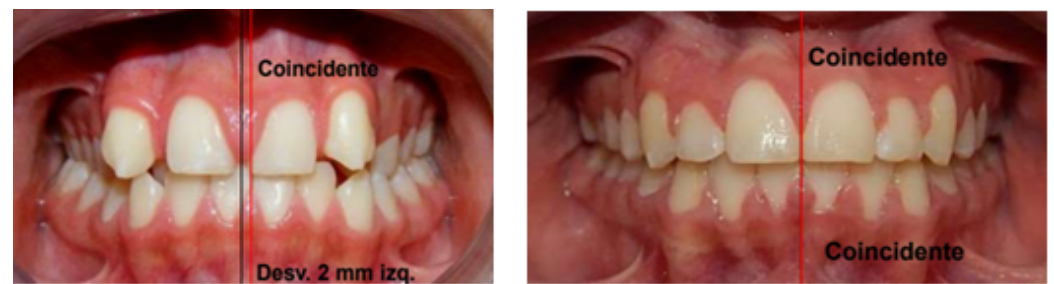

Fig. 8. Comparación Final.

revisión general del caso. En los estudios iniciales, se muestra la radiografía lateral de cráneo de inicio donde vemos el patrón esquelético clase I, normordivergencia.(Figura 1).

En el análisis intraoral de inicio: en las fotografías intraorales nos muestran: la clase II molar derecha, clase I molar izquierda, clase II canina bilateral figura, la mordida cruzada en los siguientes órganos dentarios OD 12;22;24 y 25, mordida cruzada posterior derecha (Figura 3,4), línea media inferior desviada $2 \mathrm{~mm}$ a la izquierda (Figura 5).

La forma de arco del paciente es cuadrada tanto la superior como la inferior, donde podemos observar el apiñamiento severo en superior y leve en inferior.(Figuras 6,7)

\subsection{TRATAMIENTO}

Se realizó una fase ortopédica de expansión, mediante un tornillo tipo Hyrax con el cual se logró 9 mm de expan- sión superior; para la corrección del apiñamiento superior e inferior se realizó, distalización de los caninos superiores e incorporación de órganos dentales 12 y 22; con la ayuda de arcos seccionales en segmentos postero laterales superiores.

Se obtuvo la Clase I molar derecha y se mantuvo de la Clase I molar izquierda mediante Cadenas intramaxilares ( $3 \mathrm{~s}$ a 6 s bilateral con ligadura metálica individual y en órganos dentales 11 y 21), en la fase de alineación y nivelación. Para la incorporación de los órganos dentales 12 y 22; se realizó distalización de caninos superiores, bonding en palatino de órganos dentales 12 y 22; arco adelantado superior, vestibularización mediante cuplas de órganos dentales 12 y 22, con la ayuda de una mecánica de doble arco y Tracción de OD 12 y 22 con cadenas intramaxilares e hilo elástico. Para la obtención de la máxima intercuspidación, y el adecuado paralelismo radicular, detallado, así como la oclusión funcional 


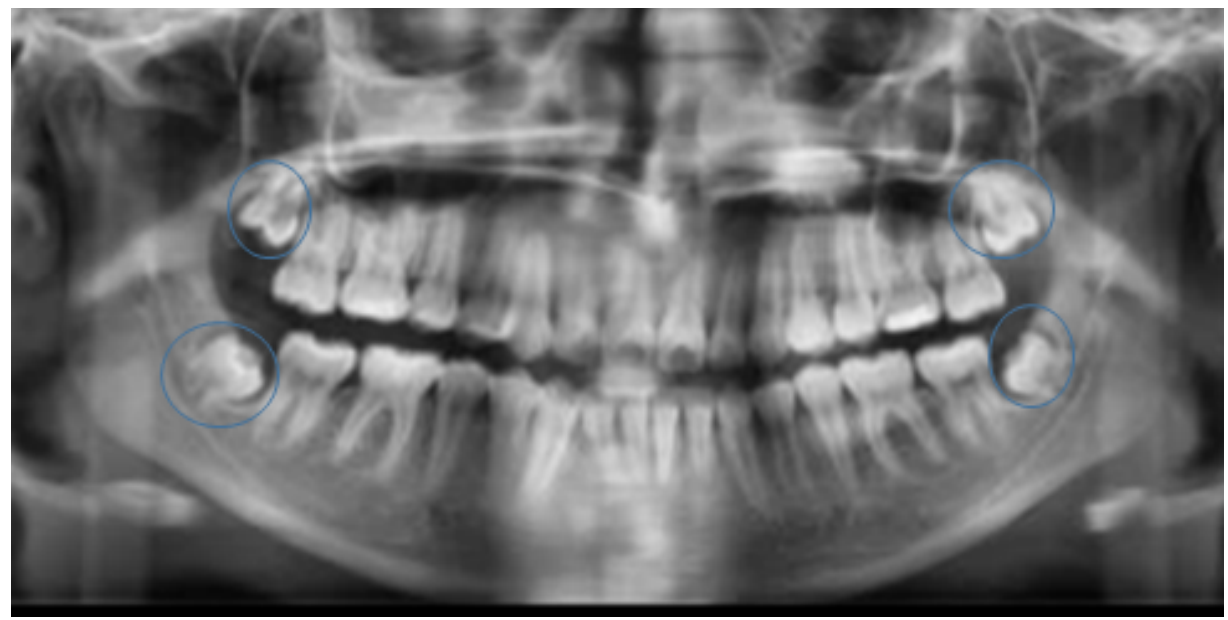

Fig. 9. Radiografía Panorámica Final.
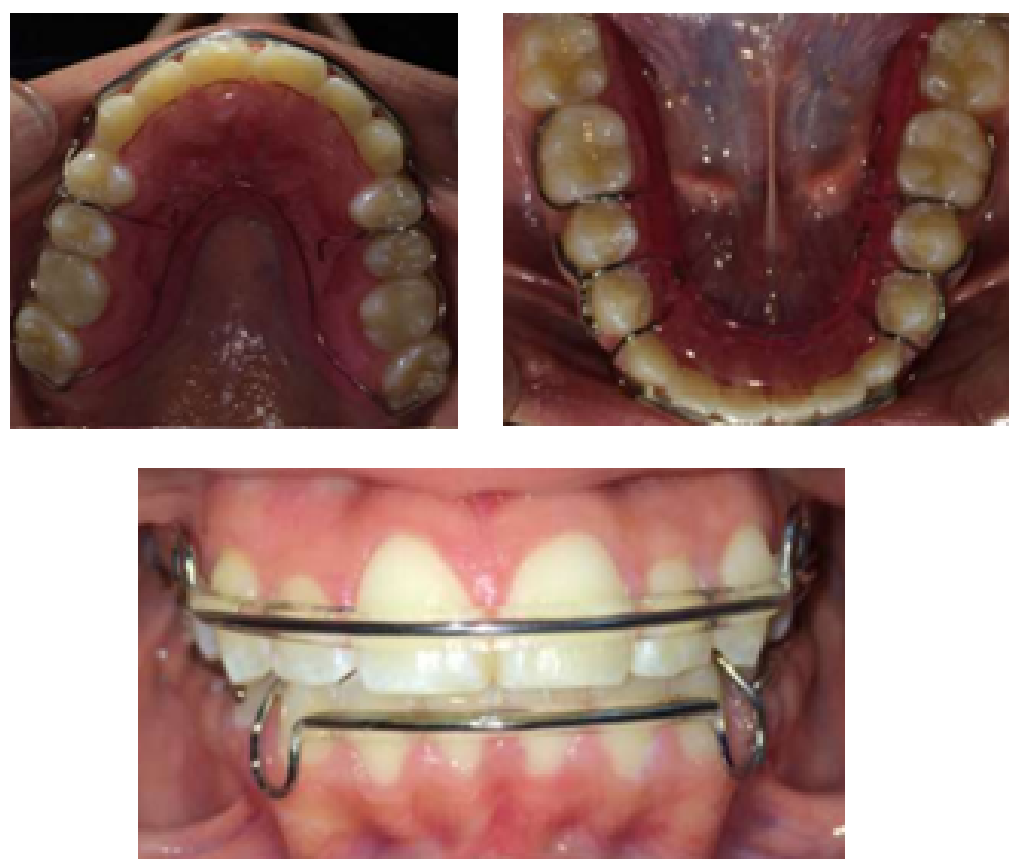

Fig. 10. Retenedores

se utilizaron arcos de acero rectangular $0,17 \times 0,25$ superior e inferior, dobleces de primer, segundo y tercer orden y elásticos en delta.

Estudios radiográficos de inicio (Figura 8) en la radiografía panorámica se pueden ver los 32 dientes presentes y que están en formación los gérmenes dentarios de los terceros molares. En los estudios de progreso se observa la notoria expansión del arco maxilar teniendo el espacio suficiente para distalización de los caninos y posterior incorporación de los órganos dentales 12 y 22.(Figura 9)

\section{RESULTADOS}

Generalmente producen una adecuada relación cráneo facial, reduciendo la necesidad de tratamientos cada vez más complejos en la dentición permanente, en la dentición infantil los problemas frecuentes son las interferencias oclusales que pueden generar maloclusiones funcionales, como las mordidas abiertas anteriores, desviaciones de la línea media, mordidas cruzadas anteriores y posteriores, y la posible alteración de la articulación temporomandibular. ${ }^{7,8}$ Por otra parte Sora recomienda que el tratamiento idóneo es buscar 
la etiología de la alteración para asegurar su estabilidad. ${ }^{9}$ Algunas asimetrías pueden ser el resultado de deflexiones mandibulares causadas por contactos prematuros durante el cierre y como consecuencia se observan mordidas cruzadas posteriores unilaterales; sin embargo, en posición de reposo la mandíbula es simétrica. ${ }^{10} \mathrm{El}$ tiempo de tratamiento de nuestro paciente fue de tres años dos meses, lográndose los objetivos planteados: una expansión de $9 \mathrm{~mm}$ con el uso de Hyrax en su fase ortopédica, se incorporaron los dientes 12 y 22; se mejoró las formas del arco, se obtuvieron las clases I bilaterales, figura 17 comparativas inicio y final del tratamiento.

\section{DISCUSIÓN}

El tratamiento precoz de la mordida cruzada posterior unilateral funcional durante la dentición mixta es extremadamente importante para la Ortodoncia porque provee un correcto posicionamiento de las bases óseas, dientes y de la articulación temporomandibular cuando el sistema estomatognático está en pleno crecimiento y desarrollo11. Es importante establecer un diagnóstico adecuado para determinar la etiología de la maloclusión, ya que una mordida cruzada de origen dental, una mordida cruzada esquelética o una combinación de las anteriores con un problema funcional.

Considerando: la edad del paciente, la magnitud del problema y la estabilidad de su corrección. ${ }^{12}$ Consideradas como alteraciones comunes, necesarias de tratar tempranamente, las maloclusiones transversales son un problema frecuente en la población joven entre el 1 y el $23 \%$, el tratamiento se realiza por medio de expansores. ${ }^{13}$ La mordida cruzada posterior es una alteración en la oclusión a la que el estomatólogo se enfrenta con frecuencia en el consultorio dental; por esta razón debe de estar capacitado para el diagnóstico precoz y tratamiento oportuno. ${ }^{2} \mathrm{El}$ desarrollo maxilar influye en el tamaño y morfología de los arcos dentarios, aunque con menos intensidad de lo que cabría esperar.

El crecimiento transversal aumenta la anchura intercanina e intermolar en distinta cuantía y amplia variabilidad individual que abarca desde niños que tienen un aumento notable hasta otros en que disminuye. Aparecen así las maloclusiones en el plano horizontal o transversal que se conocen como transversales, las cuales pueden deberse también a la fuerza compresiva de la succión como las descritas en los pacientes respiradores bucales, así como a las presiones dirigidas hacia arriba hábitos de dedo, lengua o labio, lo cual retarda la caída vertical de la porción anterior del maxilar superior. ${ }^{15}$

Las mordidas cruzadas posteriores se reportan en $15 \%$ de los pacientes. Estas pueden manifestarse en edad prematuro, y no siempre se autocorrige; si no es tratada puede generar asimetrías craneofaciales. ${ }^{15}$ En este caso realizado en el CESO, la fase ortopédica de expansión, mediante un 12 tornillo tipo Hyrax, se logró la expansión superior $9 \mathrm{~mm}$ descruzando así la mordida cruzada posterior cumpliendo con el objetivo principal en nuestro plan de tratamiento.

\section{CONCLUSIÓN}

Un diagnóstico exitoso requiere de una revisión adecuada sobre los factores etiológicos; desafortunadamente, no siempre es posible determinar con exactitud los elementos que han contribuido en la existencia de mordida cruzada posterior. Por eso debe considerarse en la fase de ortodoncia preventiva, el ser tratada con un aparato razonablemente sencillo y con esto obtener un ambiente favorable para el desarrollo normal de la oclusión.

\section{Referencias}

1 Espasa SD, J. E., Quesada Boj, J. R.,\& Ustrell i Torrent, J. M. La mordida cruzada posterior. Razones y medios para su tratamiento precoz. Anales de Odontoestomatología, 1994, núm. 4 , p. 115-122

2 Beraud O D. I., Sánchez R, M. A., Murrieta P J. F.,\& Mendoza N V. M. Prevalencia y factores de riesgo de mordida cruzada posterior en niños de 4-9 años de edad en ciudad Nezahualcóyotl. Boletín médico del Hospital Infantil de México 2004, 61(2), 141-148.

3 Ricardo RM, Comas M R B, Martínez RM R, Mok B P. Expansión rápida del maxilar con el tornillo Hyrax en un adolescente. MEDISAN [Internet]. 2015 Mar [citado 2018 Mayo 17] ; 19( 3 ): 417-421

4 Castañer PA. Ortodoncia interceptiva: Necesidad de diagnóstico y tratamiento temprano en las mordidas cruzadas transversales. Medicina Oral, Patología Oral y Cirugía Bucal (2006). (Internet), 11(2).

5 Puerta, G. Expansión rápida maxilar. Informe de un caso. Colombia Médica [Internet]. 2001;32(3):152-155. Recuperado de: http://www.redalyc.org/articulo.oa?id=28332310

6 Reyes R, M., Comas M, R. B., Martínez R, M. R.,\& Mok B, $\mathrm{P}$. Expansión rápida del maxilar con el tornillo Hyrax en un adolescente. Medisan (2015), 19(3), 417-421.

7 Cuoghi, O. A., De Mendonça, M. R., \& Miranda Z, Y. M.. Mordida cruzada posterior. Corrección y consideraciones. Caso clínico con 7 años de seguimiento. Acta Odontológica Venezolana. (2011), 49(1).

8 Quintana E, M. T.,\& Martínez B, I. Interferencias oclusales y su relación con las maloclusiones funcionales en niños con dentición mixta. Revista Médica Electrónica (2015), 32(2), $0-0$.

9 Lopera, A. M., \& Botero, P. M. Tratamiento para la corrección de mordidas cruzadas posteriores bilaterales. CES Odontología (2010), 23(1).

10 Sora, C., \& Jaramillo, P. M. Diagnóstico de las asimetrías faciales y dentales. Revista Facultad de Odontología Universidad de Antioquia (2009), 16(1 y 2).

11 Santiago CA, Díaz BR. Estudio de la dentición temporal en niños de 5 años de edad. Rev Cubana Ortod 2001:16(2)

12 Morell JA, Díaz BR. Estudio de la dentición temporal en niños de 2 a 4 años de edad. Rev Cubana Ortod 2001:16(2):11 13 Mata, J., Zambrano, F., Quirós, O., Farias, M., Rondón, S., \& Lerner, H. Expansión rápida de maxilar en maloclusiones 
transversales: revisión bibliográfica. Revista Latinoamericana de Ortodoncia y Odontopediatría (2007) [internet],

14 Cameron, A. C., Widmer, R. P., \& Martínez, A. B. (Eds.). Manual de odontología pediátrica. Harcourt Brace (2004).25(1)

15 Garbin I, A. J., Garbin S, C. A., Pantaleão dos Santos, M. R., \& Gonçalves E, P. (2007). Prevalencia de maloclusión en la dentición primaria en el municipio de Cáceres, Brasil. Revista Cubana de Estomatología, 44(1), 0-0.

Recibido: 03 de Junio de 2018.

Aceptado: 09 de Julio de 2018. 
